\title{
Influence of Various Ionic Liquids Embedded Electrospun Polymer Membrane Electrolytes on the Photovoltaic Performance of DSSC
}

\author{
Subramania Angaiah, ${ }^{1 *}$ Vignesh Murugadoss, ${ }^{1,2}$ Subasri Arunachalam, ${ }^{1,3}$ Pratheep Panneerselvam, ${ }^{1}$ and Sarathkumar Krishnan ${ }^{1}$
}

The influence of various ionic liquids such as 1,3-dimethyl imidazolium iodide (DMImI), 1-propyl-3-methyl imidazolium iodide (PMImI), 1-butyl-3-methyl imidazolium iodide (BMImI) and 1-hexyl-3-methyl imidazolium iodide (HMImI) embedded electrospun PVdF-HFP membrane electrolytes (esPMEs) on the photovoltaic performances of Dye-sensitized solar cells (DSSCs) were investigated. The influence of alkyl chain length of imidazolium and the concentration of ionic liquid electrolytes on the charge diffusion and electron transport kinetics were investigated by electrochemical impedance and Tafel polarization studies. Among the DSSCs fabricated with esPME of various ionic liquids, DSSC with 0.5M BMImI-esPME exhibited highest photoconversion efficiency of $7.08 \%$ with excellent long-term stability.

Keywords: Electrospun polymer membrane; Polymer membrane electrolyte; Ionic liquid electrolyte; Dye-sensitized solar cell

Received 21 June 2018, Accepted 25 August 2018

DOI: $10.30919 / \mathrm{es} 8 \mathrm{~d} 756$

\section{Introduction}

Dye-sensitized solar cell (DSSC) is a device that directly converts the visible light into the electrical energy. At present scenario, DSSC has much attention due to its low cost, easy and inexpensive fabrication, transparency, high photoconversion efficiency and long-term stability. ${ }^{1,2}$ Although, a photo to electric conversion efficiency of more than $13 \%$ is achieved for DSSC, ${ }^{3}$ these devices employed by volatile organic liquid electrolytes which reduce the stability and lifetime due to solvent evaporation, electrolyte leakage and electrode corrosion. In order to overcome these limitations in the liquid electrolytes for DSSCs inorganic-organic hole transport materials, room temperature ionic liquids, polymer gel electrolytes have been used as electrolytes for DSSCs. ${ }^{4-6}$ Recently, much attention on the room temperature ionic liquids (RTIL) specifically those containing the imidazolium salts due to their promising features such as negligible vapour pressure, high ionic conductivity, good thermal, electrochemical stability and wide electrochemical window. ${ }^{7,8}$ Specifically,1,3-dimethyl imidazolium iodide (DMImI), 1-propyl-3-methyl imidazolium iodide (PMImI), 1butyl-3-methyl imidazolium iodide (BMImI), 1,2-dimethyl-3-propyl imidazolium iodide (DMPmI) and 1-hexyl-3-methyl imidazolium iodide (HMImI) are the imidazolium iodide based ionic liquids widely used as electrolytes in electrochemical devices such as dye sensitized solar cell, batteries, etc. for better performance, ${ }^{9}$ because it easily solvate the redox ions and improves the ionic conductivity. However, RTIL based electrolytes show fluidity characteristics which are not

${ }^{I}$ Electrochemical Energy Research Lab, Centre for Nanoscience and Technology, Pondicherry University, Puducherry - 605 014, India

${ }^{2}$ Integrated Composites Laboratory (ICL), Department of Chemical and Biomolecular Engineering, University of Tennessee, Knoxville, Tennessee 37996, USA

${ }^{3}$ Department of Chemistry, Kalasalingam University, Krishnankovil 626126, India

*E-mail: a.subramania@gmail.com suitable for longterm stability and flexibility of DSSC. ${ }^{10}$ In order to overcome this problem, polymer gel electrolytes have been developed. Polymer gel electrolytes contain the polymer matrices embedded with different additives (RTIL, Nanoparticles, Low molecular organic gelator (LMOG), etc.,). Polymer electrolytes such as polyacrylonitrile (PAN), polyvinylidene-co-hexafluorophosphate (PVdF-HFP), polymethylmethacrylate (PMMA), polyethylene oxide (PEO) and polyaniline (PA) with different additives have been used in quasi-solid state DSSCs. ${ }^{11-15}$ Among these PVdF-HFP polymer has high electronegativity and smallest ionic radius of fluorine which leads to improved ionic conductivity, photochemically stable, good mechanical strength and flexibility. ${ }^{16}$ These polymer membranes were prepared by various techniques such as drawing, template synthesis, casting, phase separation, and electrospinning technique. Among these techniques, electrospinning technique is a simple, versatile and cost-effective approach for producing continuous ultrafine fibers and homogeneous polymer membrane with pores in micro to nano size. ${ }^{17}$ The interconnected pores give high porosity and good mechanical strength; it increases the electrolyte uptake results in the improved ionic conductivity. The ionic liquids electrolyte such as PMImI, BMImI, DMPImI and HMImI embedded electrospun polymer membrane electrolytes for DSSC have also been studied. ${ }^{18-21}$ But, there is no report on the effect of these various concentrations of imidazolium-based ionic liquid electrolytes embedded containing electrospun PVDF-HFP membrane electrolyte on the photovoltaic performance of DSSC.

Hence, in the present investigation, the influence of alkyl chain length and the concentration of various imidazolium-based ionic liquids embedded electrospun PVDF-HFP membrane electrolytes on the photovoltaic performance of DSSC were studied in detail. Each electrolyte has an important role in achieving the higher efficiency, so the optimization of the electrolyte is required to get high-performance DSSC. We examined the electrochemical behavior of various concentration of ionic liquid electrolytes. Besides, the photocurrent density-voltage $(\mathrm{J}-\mathrm{V})$ performance and stability of the DSSCs were also studied in detail. 


\section{Experimental section}

\subsection{Materials}

Ionic liquids such as DMImI, PMImI, BMImI, HMImI, Lithium iodide (LiI), Iodide $\left(\mathrm{I}_{2}\right)$, 4-tertbutyl pyridine (TBP), Acetonitrile, N719 dye were purchased from Sigma-Aldrich. N, N-dimethyl acetamide and acetone were purchased from Merck and PVdF-HFP (Kynarflex 2801) from Arkema. Platinum paste and $\mathrm{TiO}_{2}$ paste were purchased from Dyesol. All these chemicals and reagents were used without any further purifications.

\subsection{Preparation of ionic liquid electrolytes}

Table 1 shows the list of ionic liquids and their structures used in this study. Ionic liquid electrolytes were prepared at different concentrations $(0.1 \mathrm{M}, 0.3 \mathrm{M}$, and $0.5 \mathrm{M})$ by using ionic liquids such as DMImI, PMImI, BMImI and HMImI to the solution containing $0.5 \mathrm{M}$ LiI, $0.05 \mathrm{M} \mathrm{I}_{2}$ and $0.5 \mathrm{M}$ TBP in acetonitrile. Fig. 1 shows the images of the prepared ionic liquids containing electrolytes.

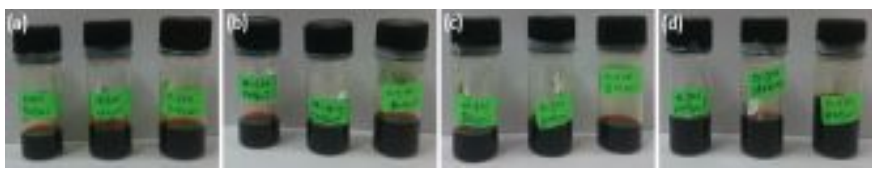

Fig. 1 Photograph of prepared ionic liquids (a) DMImI, (b) PMImI, (c) BMImI and (d) HMImI containing electrolytes.

Table 1. Ionic liquids used in this study.

\begin{tabular}{lll}
\hline Ionic liquids & $\begin{array}{l}\text { Alkyl chain } \\
\text { length }\end{array}$ \\
$\begin{array}{l}\text { 1,3-dimethyl } \\
\text { imidazolium iodide } \\
\text { (DMImI) }\end{array}$ & $\mathrm{C}_{2}$ \\
$\begin{array}{l}\text { 1-propyl-3-methyl } \\
\text { imidazolium iodide } \\
\text { (PMImI) }\end{array}$ & $\mathrm{C}_{3}$ \\
$\begin{array}{l}\text { 1-butyl-3-methyl } \\
\text { imidazolium iodide } \\
\text { (BMImI) }\end{array}$ & $\mathrm{C}_{4}$ \\
$\begin{array}{l}\text { 1-hexyl-3-methyl } \\
\text { imidazolium iodide } \\
\text { (HMImI) }\end{array}$ & $\mathrm{C}_{6}$ & $\begin{array}{l}\text { Chemical } \\
\text { formula }\end{array}$ \\
\hline
\end{tabular}

\subsection{Preparation of PVdF-HFP membrane}

The electrospun PVdF-HFP membrane was prepared by using 16 $\mathrm{wt} \%$ of the PVdF-HFP solution in a mixture of acetone/N, Ndimethyl acetamide (7: $3 \mathrm{wt} \%$ ). The resulting polymer solution was supplied to the stainless-steel syringe needle $(27 \mathrm{G})$ using a syringe pump at a flow rate of $0.5 \mathrm{~mL} / \mathrm{h}$. The electrospinning was carried out at the voltage of $19 \mathrm{kV}$ and the distance between the collector and tip of the needle was kept at $12 \mathrm{~cm}$. The resulting nanofibrous membrane was vacuum dried at $80{ }^{\circ} \mathrm{C}$ for $12 \mathrm{~h}$ to remove the residual solvent. The thickness of electrospun PVdF-HFP membrane (esPM) was reduced from about $30 \mu \mathrm{m}$ to $20 \mu \mathrm{m}$ by hot pressing at $60{ }^{\circ} \mathrm{C} .{ }^{22,23}$

The prepared esPM was soaked in the ionic liquid electrolytes comprises of $0.5 \mathrm{M} \mathrm{LiI,} 0.05 \mathrm{M} \mathrm{I}_{2}$ and $0.5 \mathrm{M}$ TBP and optimum concentration of ionic liquid in acetonitrile for $24 \mathrm{~h}$ to obtain esPMEs.

\subsection{Characterization of esPM}

The surface morphology of esPM was investigated by field emission scanning electron microscopy (FE-SEM model: JSM-JEOL 7600F). The porosity of the esPM was measured by activating the membrane with and without 1 -butanol for $2 \mathrm{~h}$. The porosity was measured by using the following equation, ${ }^{24}$

$$
\operatorname{Porosity}(\%)=\frac{\mathrm{m}_{\mathrm{a}} / \rho_{\mathrm{a}}}{\mathrm{m}_{\mathrm{a}} / \rho_{\mathrm{a}}+\mathrm{m}_{\mathrm{p}} / \rho_{\mathrm{p}}}
$$

where $m_{a}$ and $m_{p}$ are the weight of esPM after and before impregnation with 1-butanol and $\rho_{\mathrm{a}}$ and $\rho_{\mathrm{p}}$ are the density of 1butanol and the dried esPM respectively.

The electrolyte uptake (U) of the esPM can be calculated by immersing the membrane in an electrolyte solution containing $0.5 \mathrm{M}$ LiI, $0.05 \mathrm{M} \mathrm{I}_{2}$ and $0.5 \mathrm{M}$ TBP in acetonitrile for $24 \mathrm{~h}$. The electrolyte uptake (U) was measured by using the formula, ${ }^{25}$

$$
\mathrm{U}(\%)=\left[\left(\mathrm{m}-\mathrm{m}_{\mathrm{o}}\right) / \mathrm{m}_{\mathrm{o}}\right] \times 100
$$

where $\mathrm{m}$ and $\mathrm{m}_{\mathrm{o}}$ are the mass of wet and dry membranes respectively.

\subsection{Electrochemical characterizations}

The effects of various concentrations $(0.1 \mathrm{M}, 0.3 \mathrm{M}$ and $0.5 \mathrm{M})$ of ionic liquids (DMImI, PMImI, BMImI and HMImI) containing electrolytes on the interfacial resistance at the electrode/electrolyte interface and the diffusion coefficient of $\mathrm{I}_{3}^{-} \mathrm{I}_{3}^{-}$were determined by AC impedance studies using symmetric cell comprising FTO/Pt/electrolyte/Pt/FTO. The frequency limit was set between $1 \mathrm{mHz}$ and $100 \mathrm{kHz}$ with an applied AC amplitude of $10 \mathrm{mV}$. Tafel polarization studies were carried out to confirm further the electrocatalytic activity of electrolyte, by using symmetric cell comprising FTO/Pt/electrolyte/Pt/FTO. Tafel polarization was performed by using an Electrochemical workstation (Biologic VSP) at a scan rate of $50 \mathrm{mV} \mathrm{s}^{-1}$ in the voltage range of $-2 \mathrm{~V}$ to $+2 \mathrm{~V}$. In Tafel curves, a larger slope is the anodic or cathodic branch indicates a higher exchange current density $\left(J_{0}\right)$. The $J_{0}$ is inversely proportional to $\mathrm{R}_{\mathrm{ct}}$ and it is related by the following equation;

$$
J_{o}=\frac{R T}{n F R_{c t}}
$$

where $\mathrm{R}$ is a gas constant, $\mathrm{T}$ is an absolute temperature, $\mathrm{n}$ is the number of electrons involving in the charge transfer process and $\mathrm{F}$ is a Faraday constant and $R_{c t}$ is the charge transfer resistance.

The diffusion coefficient was calculated from the following equation;

$$
J_{\text {lim }}=\frac{2 n F C D_{n}}{d}
$$

where, $\mathrm{n}$ is the number of electrons contributing to the charge transfer process, $\mathrm{F}$ is a Faraday constant, $D_{n}$ is a diffusion coefficient of $\mathrm{I}^{-} / \mathrm{I}_{3}{ }^{-}$and $\mathrm{d}$ is a distance between the electrodes in the symmetric cell. The $J_{\lim }$ values are obtained from the intersection of the cathodic branch with y-axis at a high potential in Tafel curves. ${ }^{26}$ The Nyquist and Tafel plots of the symmetrical dummy cells were recorded at open circuit potential under dark conditions. All the symmetrical dummy cells were assembled by using two identical platinum coated ITO glass substrates with the ionic liquids 
containing liquid electrolyte to study the charge transfer resistance and diffusion coefficient of the redox couple. The active surface is $0.20 \mathrm{~cm}^{2}$ for all the symmetrical dummy cells.

The Ionic conductivity of the prepared esPME was measured at $25{ }^{\circ} \mathrm{C}$ using electrochemical workstation (Biologic VSP) from AC impedance spectroscopy at a frequency range between $1 \mathrm{mHz}$ to 100 $\mathrm{kHz}$ at an amplitude of $10 \mathrm{mV}$, by sandwiching the same between two stainless steel blocking electrodes. The ionic conductivity was calculated by using the following equation, ${ }^{27}$

$$
\sigma=\frac{1}{\mathrm{RA}}
$$

where 1 is the thickness of the membrane measured by the thickness measuring gauge, $\mathrm{R}$ is the high-frequency intercept of the impedance plot and A is the area of esPME. The area and thickness of all the esPMEs are $1 \mathrm{~cm}^{2}$ and $20 \mu \mathrm{m}$, respectively.

\subsection{Fabrication of DSSC}

The FTO conducting glass was cleaned with the help of detergent for $20 \mathrm{~min}$ and followed by acetone and ethanol for 20 min using an ultrasonic bath. The cleaned FTO was dried in an air oven at $60{ }^{\circ} \mathrm{C}$ for $2 \mathrm{~h}$. The $\mathrm{TiO}_{2}$ paste was applied on the FTO glass by Doctor Blade method. The $\mathrm{TiO}_{2}$ photoanode was sintered at $450{ }^{\circ} \mathrm{C}$ for 30 min to get nanocrystalline $\mathrm{TiO}_{2}$ photoanode. The same procedure is carried out for the preparation of $\mathrm{Pt}$ counter electrode. The $\mathrm{TiO}_{2}$ photoanode was soaked in the N719 dye for $24 \mathrm{~h}$ at the dark condition and the dye-sensitized $\mathrm{TiO}_{2}$ photoanode rinsed with ethanol to clean the unabsorbed dye on the $\mathrm{TiO}_{2}$ photoanode. ${ }^{28,29}$ The DSSCs were then fabricated by sandwiching the prepared esPME obtained by activating esPM by ionic liquid electrolytes containing an optimum concentration of various ionic liquid electrolytes $(0.3 \mathrm{M}$ DMImI, 0.5 M PMImI, 0.5 M BMImI or 0.3 M HMImI in between the dye-sensitized $\mathrm{TiO}_{2}$ photoanode and $\mathrm{Pt}$ counter electrode. The sandwiched cells were stacked together by sealing with surlyn (60 $\mu \mathrm{m})$ spacer hot pressed at $110{ }^{\circ} \mathrm{C}$ by hot press thermoplastic sealer.

\subsection{Photovoltaic performance and Stability studies of DSSCs}

The photocurrent density-voltage $(J-\mathrm{V})$ characteristics of the fabricated DSSCs with an active area of $0.5 \mathrm{~cm}^{2}$ were measured by computer-controlled digital source meter (Keithley Model: 2420) and controlled by solar simulator (Newport Oriel instruments Model: 67005) with AM 1.5 filter coupled with $450 \mathrm{~W}$ Xenon lamp was used to illuminate the DSSC at $1000 \mathrm{~W} \mathrm{~m}^{-2}$. The incident light intensity was calibrated by using a reference silicon solar cell before the experiment. Hermetically sealed cells were stored at ambient temperature and the electrochemical measurements were carried out for every $48 \mathrm{~h}$ to study the long-term stability test of the device. ${ }^{30}$ The photoelectrochemical parameters such as fill factor (FF) and the incident photon to current conversion efficiency $(\eta)$ were measured using the following equation, ${ }^{31}$

$$
\begin{gathered}
\eta(\%)=\frac{\mathrm{V}_{\max } \cdot \mathrm{J}_{\max }}{\mathrm{P}_{\text {in }}} \times 100=\frac{\mathrm{V}_{o c} \cdot \mathrm{J}_{o c} \mathrm{FF}}{\mathrm{P}_{\text {in }}} \times 100 \\
\mathrm{FF}=\frac{\mathrm{V}_{\max } \cdot \mathrm{J}_{\max }}{\mathrm{V}_{o c} \cdot \mathrm{J}_{s c}}
\end{gathered}
$$

where $J_{s c}$ is the short-circuit current density $\left(\mathrm{mA} \mathrm{cm}^{-2}\right), V_{o c}$ is the open-circuit voltage $(\mathrm{V}), \mathrm{P}_{\mathrm{in}}$ is the incident light power $(\mathrm{mW} \mathrm{cm})$, and $J_{\max }$ and $\mathrm{V}_{\max }$ are the current density $\left(\mathrm{mAcm}^{-2}\right)$ and voltage $(V)$ in the $J$ - $V$ curves, respectively, at the point of maximum power output.

\section{Results and discussion}

\subsection{Electrochemical measurements of ILEs}

Figs. 2 shows the Nyquist plot of the symmetry cells with various concentrations of four kinds of ionic liquids containing electrolytes. Their insets show the equivalent circuit used to analyze the impedance spectra fitted by using $E C$ lab software. Here, $R_{\mathrm{s}}$ is the series resistance representing the resistance of the substrate in the higher frequency region, $R_{\mathrm{ct}}$ is the charge transfer resistance at the electrode-electrolyte interface, and $C_{\mathrm{d} 1}$ is its corresponding capacitance, and $Z_{\mathrm{w}}$ is the Nernst diffusion impedance in the lower frequency region ${ }^{32}$. The effect of various concentrations of ionic liquids and their alkyl chain length on the charge transfer is investigated by electrochemical impedance and Tafel polarization studies. The measured values of $R_{\mathrm{s}}$ and $R_{\mathrm{ct}}$ obtained from $E C$ lab software are given in Table 2.

The increase in the concentration of ionic liquid increases the number of mobile charge carriers which can lead to the enhancement of the ionic conductivity. Beyond an optimum concentration, the aggregation of ions decreases the density of ions by the formation of ion clusters. It results in the decrease of ionic conductivity. The optimum concentrations are found to be $0.3 \mathrm{M}$ for DMImI \& HMImI and $0.5 \mathrm{M}$ for PMImI \& BMImI. In our previous studies, we observed that $0.7 \mathrm{M}$ BMImI exhibits lower ionic conductivity than that at the concentration of $0.5 \mathrm{M} \mathrm{BMImI}{ }^{20}$ Hence, we carried out the experiments up to $0.5 \mathrm{M}$ for all the ionic liquids.
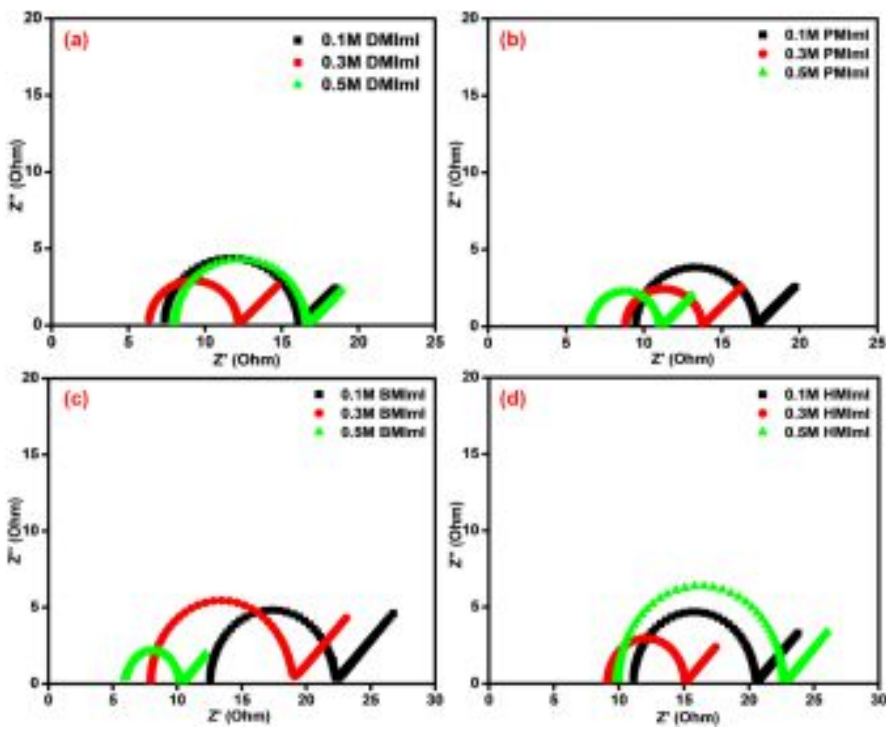

Figure 2 Nyquist plot of the symmetry cells with various concentrations of four kinds of ionic liquids containing electrolytes.

It is observed from the Fig. 4a and Table 2, among the various imidazolium-based ionic liquid electrolytes, $0.5 \mathrm{M}$ BMImI shows less charge transfer resistance. This is because that the viscosity of imidazolium iodide increases with increasing the length of the alkyl chain and thereby decreases the ionic conductivity., Increasing the length of alkyl chain from $C_{4}$ to $C_{6}$ increases the charge transfer resistance due to its viscosity because of Vander Waals force between the alkyl chains that induce the accumulation of cations showing glue-like nature that hinders the free ion mobility., Even 
though the alkyl chain length less than $C_{4}$ it facilitates the faster triiodide diffusion in the electrolyte, the less ion dissociation obstructs their ionic conductivity. Thus, the BMImI with chain length $C_{4}$ at the optimum concentration of $0.5 \mathrm{M}$ exhibits comparatively fewer $R_{\mathrm{s}}$ and $R_{\mathrm{ct}}$ values than that of other concentration of ionic liquids.

Figs. 3 shows the Tafel polarization curves for the symmetry cells with the various concentrations of four kinds of ionic liquids containing electrolytes. The Tafel polarization studies describe the ionic diffusivity $\left(\mathrm{D}_{\mathrm{n}}\right)$ in the electrolytes. The $J_{0}$ value obtained from Eq. (3) describes that the higher $J_{\mathrm{o}}$ values indicate the low charge transfer resistance between the electrode/electrolyte interface. ${ }^{37}$ The ionic diffusivity in the electrolyte is measured by Eq. (4) by using the $J_{\lim }$ value from the Tafel polarization curves. Higher $J_{\lim }$ value indicates the high diffusion of ions in the electrolyte which improves the ionic conductivity.

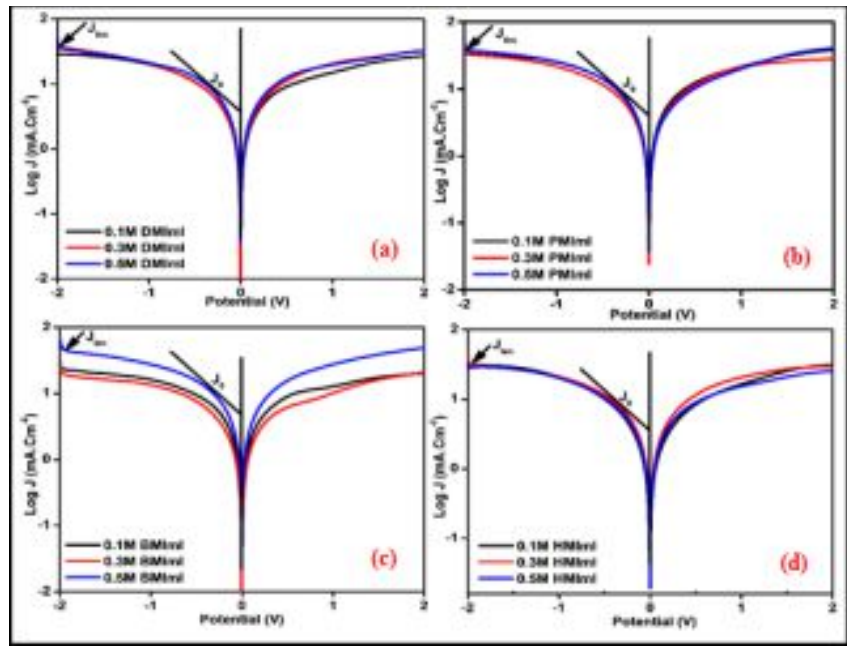

Figs. 3 Tafel polarization curves of the symmetry cells with various concentrations of four kinds of ionic liquids containing electrolytes.

Tafel polarization parameters calculated from the Eq. (3) and (4) for the symmetric cells fabricated with various concentrations of four kinds of ionic liquids containing electrolytes are given in Table 2. It is observed that it all the four ionic liquids, the diffusion kinetics of redox species in the electrolyte decreases beyond the optimum concentration. This may be due to the accumulation of excess cations, which may increase the charge transfer resistance. The diffusion kinetics of the ionic liquid electrolytes with respect to their alkyl chain length can be understood from their viscous behavior through Coulombic and VanderWaals interaction. At smaller alkyl chain length, the Coulombic force between the anions and cations are strong that retard the dissociation. At higher chain length, the association effect due to the Vander Waals interaction between the alkyl imidazolium cations obstructs the mass transport.

The investigation of the electrochemical property of electrolytes obtained from the Tafel polarization studies is well consistent with the EIS measurements. The optimum concentration of various ionic liquids such as $0.3 \mathrm{M}$ DMImI, 0.5 M PMImI, 0.5 M BMImI and 0.3 M HMImI were deduced from the electrochemical investigations. Among them, 0.5 M BMImI ionic liquid containing electrolyte exhibits lower charge transfer resistance and higher diffusion kinetics for $\mathrm{I}^{-} / \mathrm{I}_{3}^{-}$redox species. The Nyquist plots and Tafel polarization curves for the optimum concentration of four ionic liquid electrolytes are shown in
Figs. 4(a\&b).
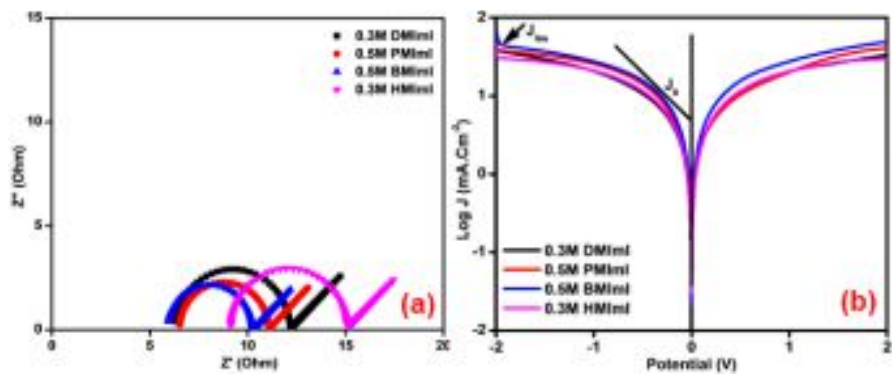

Fig. 4 (a) Nyquist plots and (b) Tafel polarization curves of the symmetric cells fabricated with the optimum concentration of various ionic liquids containing electrolytes.

Table 2. Electrochemical impedance and Tafel polarization parameters of the symmetric cells fabricated with various concentrations of four kinds of ionic liquids containing electrolytes.

\begin{tabular}{|c|c|c|c|c|c|}
\hline \multirow[b]{2}{*}{$\begin{array}{c}\text { Ionic } \\
\text { Liquid }\end{array}$} & \multirow[b]{2}{*}{$\begin{array}{l}\text { Conc. of } \\
\text { ionic } \\
\text { liquid }\end{array}$} & \multicolumn{2}{|c|}{ AC impedance parameters } & \multicolumn{2}{|c|}{ Tafel polarization parameters } \\
\hline & & $\begin{array}{c}\text { Series } \\
\text { resistance } \\
\left(\mathrm{R}_{\mathrm{s}}\right) \\
\mathrm{Ohm}\end{array}$ & $\begin{array}{c}\text { Charge transfer } \\
\text { resistance } \\
\left(\mathrm{R}_{\mathrm{ct}}\right) \\
\mathrm{Ohm}\end{array}$ & $\begin{array}{c}\text { Limiting } \\
\text { current } \\
\text { density }\left(\mathrm{J}_{\lim }\right) \\
\mathrm{mA} \cdot \mathrm{cm}^{-2}\end{array}$ & $\begin{array}{c}\text { Diffusion coefficient } \\
\text { of } \mathrm{I}^{-} \\
\times 10^{-5} \mathrm{~cm}^{2} \mathrm{~s}^{-1}\end{array}$ \\
\hline \multirow{3}{*}{ DMImI } & $0.1 \mathrm{M}$ & 7.37 & 8.712 & 1.4628 & 2.275 \\
\hline & $0.3 \mathrm{M}$ & 6.33 & 5.808 & 1.5931 & 2.476 \\
\hline & $0.5 \mathrm{M}$ & 7.99 & 8.607 & 1.5785 & 2.454 \\
\hline \multirow{3}{*}{ PMImI } & $0.1 \mathrm{M}$ & 9.48 & 7.658 & 1.5447 & 2.401 \\
\hline & $0.3 \mathrm{M}$ & 8.82 & 4.887 & 1.5517 & 2.412 \\
\hline & $0.5 \mathrm{M}$ & 6.53 & 4.537 & 1.5969 & 2.482 \\
\hline \multirow{3}{*}{ BMImI } & $0.1 \mathrm{M}$ & 12.55 & 9.624 & 1.3921 & 2.164 \\
\hline & $0.3 \mathrm{M}$ & 7.94 & 10.85 & 1.3176 & 2.048 \\
\hline & $0.5 \mathrm{M}$ & 5.95 & 4.321 & 1.6747 & 2.603 \\
\hline \multirow{3}{*}{ HMImI } & $0.1 \mathrm{M}$ & 13.12 & 9.357 & 1.4912 & 2.318 \\
\hline & $0.3 \mathrm{M}$ & 9.14 & 5.910 & 1.4962 & 2.326 \\
\hline & $0.5 \mathrm{M}$ & 9.89 & 12.73 & 1.4533 & 2.259 \\
\hline
\end{tabular}

\subsection{Morphological and Structural Properties of esPM}

It is reported in the earlier literature that the efficiency of liquid electrolyte decreased immediately due to the solvent evaporation and leakage. ${ }^{38}$ Even though ionic liquid suppresses the evaporation of the solvent, it has fluidic property. In order to further improve the stability of the DSSC, ionic liquid embedded electrospun PVdF-HFP membrane electrolyte (esPME) was used as an electrolyte in this study.

Fig. 5a shows the FE-SEM image of the prepared electrospun PVdF-HFP membrane (esPM). The esPM comprise nanofibers with a uniform size distribution and well-defined structure. The average fiber diameter of esPM is $\sim 300-400 \mathrm{~nm}$. The polymer matrix of esPM comprises of a three-dimensional network structure with high porosity. The pores are fully interconnected and capable of holding a huge amount of ionic liquid containing the electrolyte. The esPM has maximum porosity and electrolyte uptake of $87 \%$ and $335 \%$ respectively. The electrospun membrane has good mechanical strength due to its three-dimensional network structure with crosslinking points. ${ }^{39}$ 


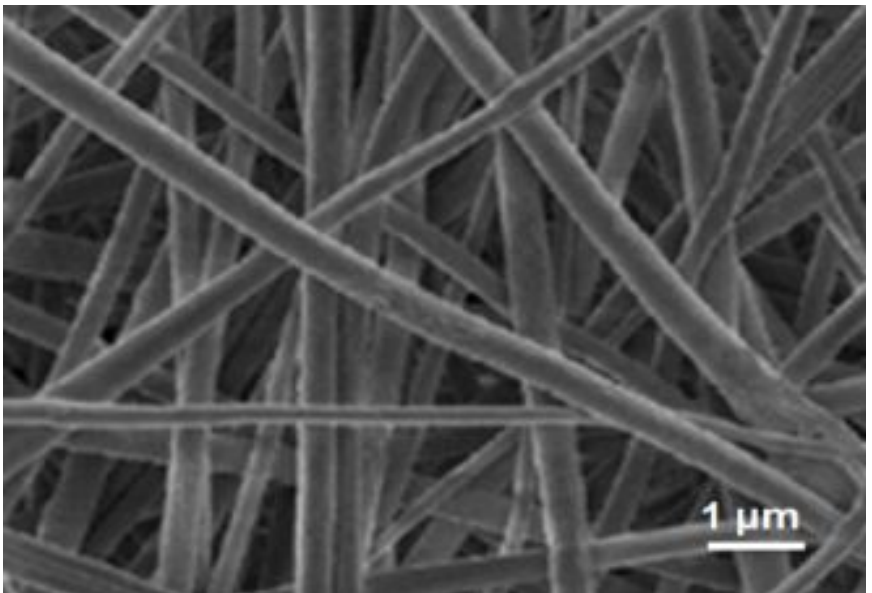

Fig. 5a FE-SEM image of the electrospun PVDF-HFP polymer membrane.

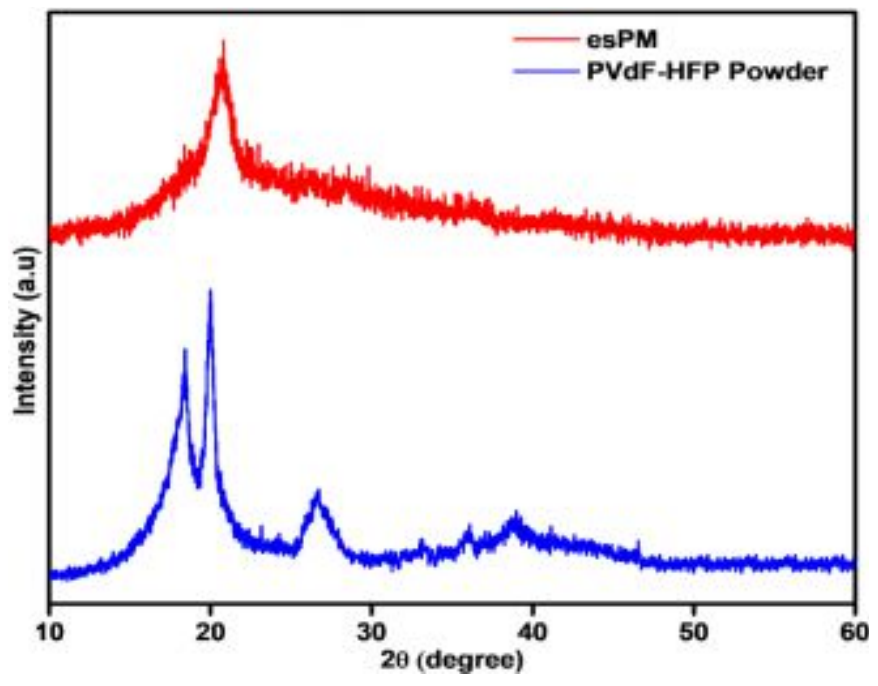

Fig. 5b XRD patterns of PVdF-HFP powder and electrospun PVdF-HFP polymer membrane (esPM).

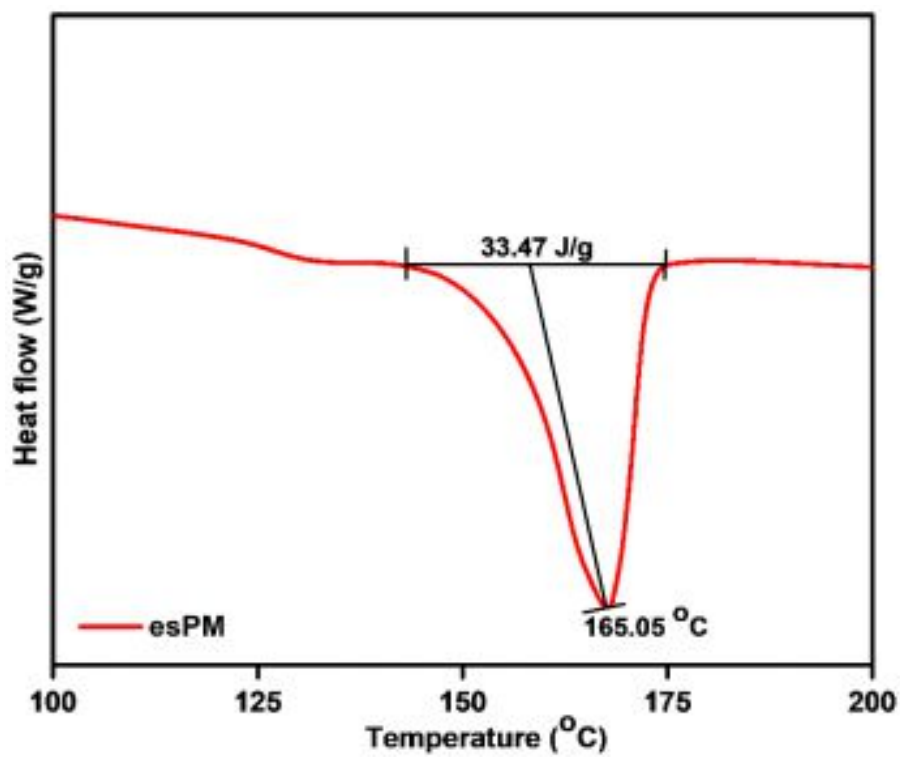

Fig. 5c DSC thermogram of the electrospun PVdF-HFP membrane (esPM).
Fig. $5 b$ shows the XRD patterns of the PVdF-HFP powder and electrospun PVdF-HFP membrane (esPM). The characteristic peaks at $18.28^{\circ}$ and $20.08^{\circ}$, correspond to (100) and (020) crystalline planes of PVdF-HFP polymer powder confirm its semi-crystalline nature. It can also be observed from the XRD pattern of esPM that the peak at $18.28^{\circ}$ gets disappeared and the peak at $20.08^{\circ}$ becomes relatively weak and broad compared to that of the sharp peaks of PVdF-HFP powder. This indicates that the crystalline microstructure of the electrospun PVdF-HFP fibers was not well developed, suggesting a decrease in the degree of crystallinity of the polymer matrix. The crystallinity of esPM is lower than that of PVdF-HFP powder, because of the rapid solidification of PVdF-HFP chains during electrospinning. ${ }^{40}$

Further, the decrease in the degree of crystallinity of esPM is confirmed by DSC analysis. Fig. 5c shows the DSC thermograms of esPM in the temperature range between $100{ }^{\circ} \mathrm{C}$ and $200{ }^{\circ} \mathrm{C}$. Considering the melting enthalpy of $100 \%$ crystalline PVdF-HFP powder $\left(\triangle H_{\mathrm{m}}^{*}\right)$ as $104.7 \mathrm{~J} \mathrm{~g}^{-1}$, the crystallinity $\left(\mathrm{X}_{\mathrm{c}}\right)$ of the esPM is calculated from the melting enthalpy of the esPM $\left(\triangle H_{\mathrm{m}}^{\mathrm{sample}}=33.47\right.$ $\mathrm{J.g}^{-1}$ ) (Fig. 5c) using the following equation, ${ }^{41}$

$$
X_{c}=\frac{\Delta H_{m}^{\text {sample }}}{\Delta H_{m}^{*}} \times 100(\%)
$$

The crystallinity of esPM is found to be $31.96 \%$ which is consistent with the XRD result of decreasing crystallinity. The decrease in crystallinity of the polymer matrix promotes intra- and inter-chain hopping of the ions, which improve the ionic conductivity of esPME. ${ }^{41}$

\subsection{Ionic conductivity studies of esPME}

Fig. 6 shows the Nyquist plots of the optimum concentration of various ionic liquids (0.3 M DMImI, 0.5 M PMImI, 0.5 M BMImI and $0.3 \mathrm{M}$ HMImI) embedded esPME. It can be observed that the obtained AC impedance spectra are capacitive dominant rather than the inductive effects and hence represented as Nyquist plots. ${ }^{42}$ Here, Z' (X-axis) represents the real part $[\operatorname{Re}(\mathrm{Z})]$ and $\mathrm{Z}$ ' (Y-axis) represents the imaginary part of the impedance $[-\operatorname{Im}(Z)] .^{43}$ The values of ionic conductivity given in Table 3 shows the trend of 0.5 M BMImI $\left(6.648 \times 10^{-3}{\mathrm{~S} . \mathrm{cm}^{-1}}^{-1}>0.3 \mathrm{MPMImI}\left(5.653 \times 10^{-3}{\mathrm{~S} . \mathrm{cm}^{-1}}^{-5}>\right.\right.$ $0.3 \mathrm{M}$ DMImI $\left(4.584 \times 10^{-3}{\mathrm{~S} . \mathrm{cm}^{-1}}^{-1}>0.3 \mathrm{M} \mathrm{HMImI}\left(3.892 \times 10^{-3}\right.\right.$ $\left.\mathrm{S} . \mathrm{cm}^{-1}\right)$. It infers that the ionic conductivity strongly related to the interfacial resistances. The ionic conductivity is increases with increase in the alkyl chain from $\mathrm{C}_{2}$ to $\mathrm{C}_{4}$, which is attributed to the increase in the interaction of ionic liquid with the polymer host (esPM). This interaction provides the effect of plasticization, which leads to the enhanced flexibility in the polymer backbone, thereby increases the ionic conductivity. On increasing chain length, more interaction of the ionic liquid with the polymer host can induce the additional crystalline phase in the polymer backbone. This additional crystalline phase may hinder the ionic motion. Thus, beyond $\mathrm{C}_{4}$, the ionic conductivity decreases for ionic liquid with the chain length of $\mathrm{C}_{6}$ (HMImI). These results are well consistent with that are obtained from electrochemical impedance and Tafel polarization studies measurements for ionic liquids embedded esPMEs. ${ }^{20}$ 


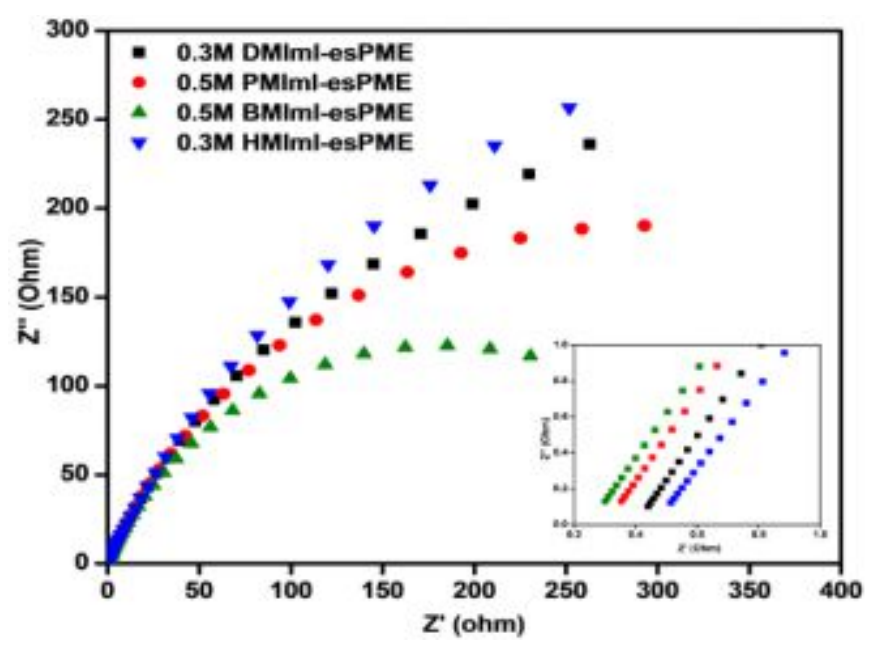

Fig. 6 Nyquist plots for the optimum concentration of various ionic liquids embedded esPMEs.

Table 3. Ionic conductivity values of the esPME embedded with the optimum concentration of various ionic liquids

\begin{tabular}{lc}
\hline Electrolyte system & $\begin{array}{r}\text { Ionic conductivity } \\
\left(\times 10^{-3} \mathrm{~S} \mathrm{~cm}^{-1}\right)\end{array}$ \\
\hline $0.3 \mathrm{M}$ DMImI - esPME & 4.584 \\
$0.5 \mathrm{M}$ PMImI - esPME & 5.653 \\
$0.5 \mathrm{M}$ BMImI - esPME & 6.648 \\
$0.3 \mathrm{M} \mathrm{HMImI}$ - esPME & 3.892 \\
\hline
\end{tabular}

\subsection{Photovoltaic performance studies}

The photocurrent density-voltage (J-V) curves of the fabricated DSSCs based on $0.5 \mathrm{M}$ BMImI-LE and various ionic liquids embedded esPMEs obtained at a light intensity of $100 \mathrm{mWcm}^{-2}$ under standard global AM 1.5 irradiation are shown in Fig. 7 and their corresponding photovoltaic parameters are displayed in Table 4.

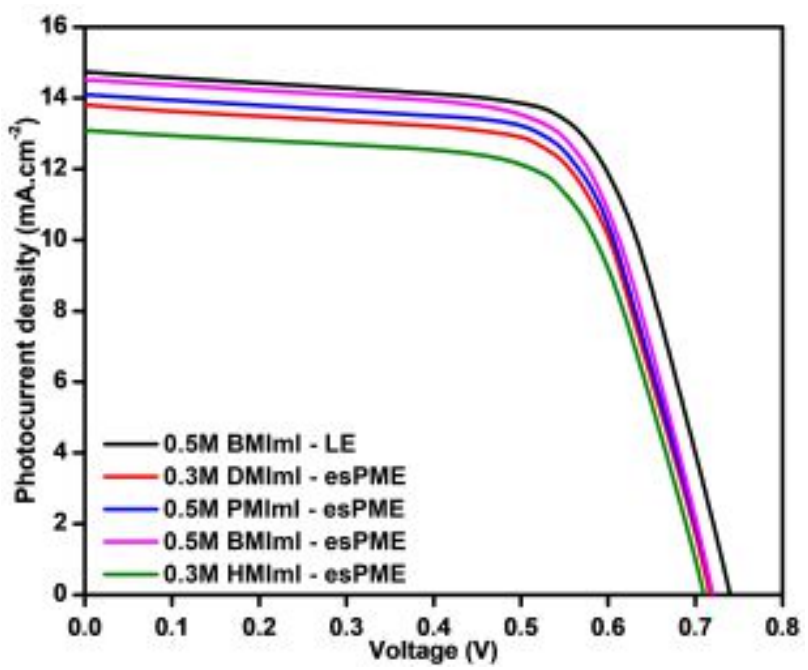

Fig. 7 Photocurrent density-voltage (J-V) curves for DSSCs fabricated with $0.5 \mathrm{M}$ BMImI-LE and optimum concentration of various ionic liquids embedded esPMEs.
Table 4. Photovoltaic performance of DSSC sfabricated $0.5 \mathrm{M}$ BMImI - LE and various ionic liquids embedded esPMEs.

\begin{tabular}{lcccc}
\hline \multicolumn{1}{c}{ Electrolyte } & $\begin{array}{c}\mathrm{J}_{\mathrm{sc}} \\
\left(\mathrm{mA} \mathrm{cm}^{-2}\right)\end{array}$ & $\begin{array}{c}\mathrm{V}_{\mathrm{oc}} \\
(\mathrm{V})\end{array}$ & $\mathrm{FF}$ & $\begin{array}{c}\text { Efficiency } \\
(\%)\end{array}$ \\
\hline 0.5M BMImI - LE & 14.73 & 0.74 & 0.682 & 7.43 \\
0.3M DMImI- esPME & 13.81 & 0.72 & 0.672 & 6.68 \\
0.5M PMImI- esPME & 14.09 & 0.72 & 0.677 & 6.86 \\
0.5M BMImI - esPME & 14.52 & 0.72 & 0.678 & 7.08 \\
0.3M HMImI- esPME & 13.09 & 0.71 & 0.672 & 6.24 \\
\hline
\end{tabular}

Both $J_{s c}$ and FF were influenced by the ionic conductivity and the diffusion of ionic species. It is reported that imidazolium iodide with higher alkyl chain length form multi-layer on $\mathrm{TiO}_{2}$ surface by adsorption due to the Vander Waals force that suppresses the backelectron transfer thereby increases the electron lifetime and decrease the recombination. At lower alkyl chain length, the chance of multilayer adsorption on the imidazolium cation is less which may decrease the electron lifetime, thereby decreases the short-circuit photocurrent density. Even though, the higher alkyl chain length increases the electron lifetime and also decreases in the diffusion coefficient of $\mathrm{I}_{3}$ resulting from high viscosity that decreases the fill factor and short circuit ${ }^{20}$. Thus, the BMImI-containing esPME has higher ionic conductivity and diffusion coefficient of $\mathrm{I}^{-} / \mathrm{I}_{3}^{-}$than other ionic liquids such as DMImI, PMImI, and HMImI embedded esPMEs. Fig. 8 shows the photo-conversion efficiency of DSSC verses the ionic conductivity of the esPMEs embedded with the optimum concentration of various ionic liquids. The DSSC fabricated with 0.5M BMImI-esPME exhibits PCE of $7.08 \%$, which is close to that of DSSC fabricated with 0.5M BMImI-LE (7.43\%).

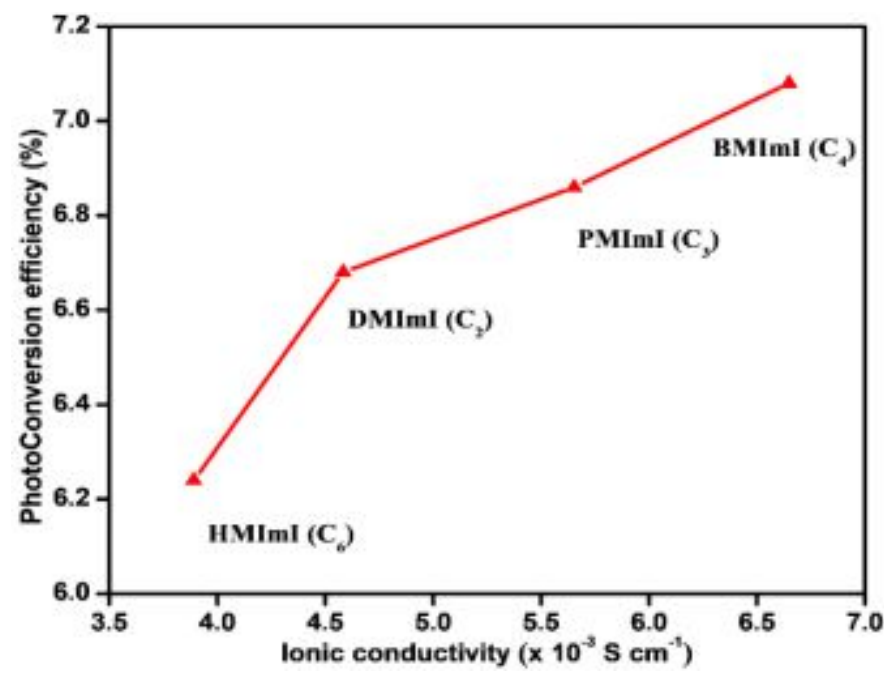

Fig. 8 Photoconversion efficiency of DSSCs fabricated with the optimum concentration of various ionic liquids embedded esPMEs with respect to their ionic conductivity.

\subsection{Stability studies}

The J-V curves of DSSCs employing 0.5 M BMImI-esPME and 0.5 M BMImI-LE were recorded over a period of 30 days with the regular interval of time and their corresponding light to electricity conversion efficiency are shown in Fig. 9. The cell efficiency was recorded for every $48 \mathrm{~h}$ after storage in the dark at ambient temperature. ${ }^{39}$ 


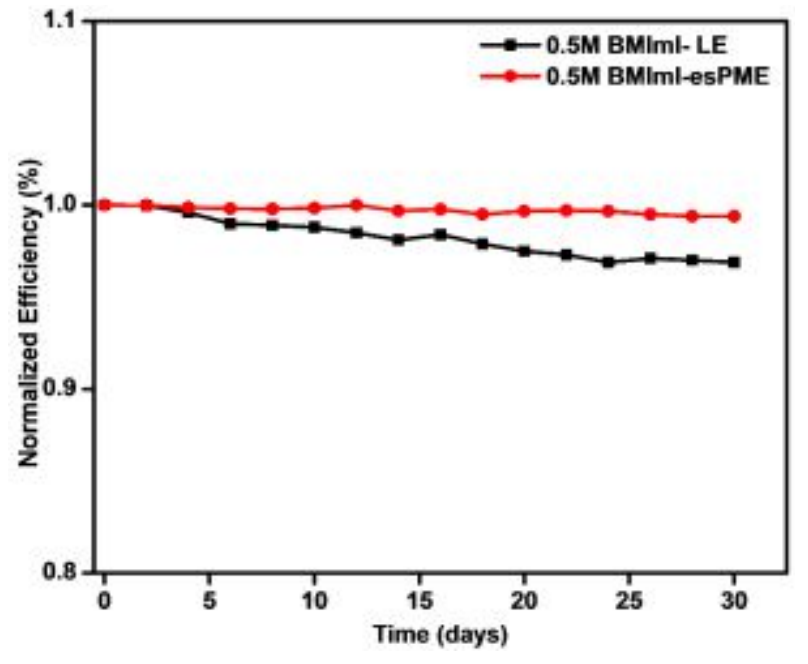

Fig. 9 Normalized light to electricity conversion efficiency of DSSCs containing 0.5 M BMImI-LE and 0.5 M BMImI-esPME.

It is observed that the normalized efficiency of DSSC fabricated with $0.5 \mathrm{M}$ BMImI-LE decreased gradually due to the solvent evaporation, whereas the same DSSC fabricated with $0.5 \mathrm{M}$ BMImI-esPME retains $99 \%$ of its initial efficiency value even after 30 days. This is because of the interconnected pore structure of esPM, which ensures the retention of the electrolyte and prevent the leakage/evaporation of the electrolyte. .4,45 $^{-10}$

\section{Conclusions}

This work highlighted the effect of alkyl chain length of different imidazolium-based ionic liquids and their concentrations in liquid electrolyte on the electrochemical measurements such as Electrochemical impedance spectroscopy and Tafel polarization studies for DSSC applications. The influence of the alkyl chain length of different ionic liquids and their concentrations on the charge transfer, mass transfer and diffusion coefficients of $\mathrm{I}^{-} / \mathrm{I}_{3}^{-}$for DSSC applications are illustrated based on their viscous behavior through Coulombic and Vander Waals interaction. Further, the optimized ionic liquids are imbibed into the electrospun PVdF-HFP membrane are prepared to improve the stability of DSSC. The ionic conductivity values of esPMEs are calculated from AC impedance spectroscopy. 0.5 M BMImI-esPME exhibited maximum ionic conductivity of about $6.658 \times 10^{-3} \mathrm{~S} . c m^{-1}$ than that of other ionic liquids. The DSSC fabricated with $0.5 \mathrm{M}$ BMImI-esPME exhibits the photoconversion efficiency ( $7.08 \%$ ) higher than that of DSSC fabricated with esPME containing other ionic liquid electrolytes and considerably superior long-term stability than the same fabricated with the $0.5 \mathrm{M}$ BMImILE.

\section{Conflict of interest}

There are no conflicts to declare.

\section{Acknowledgements}

One of the authors, Dr.AS gratefully acknowledge the Council of Scientific and Industrial Research (CSIR), New Delhi (Ref. No.01/2810/14/EMR-II) for the financial support. Mr. MV grateful to the Indo-US Science and Technology Forum (IUSSTF), Department of Science and Technology (DST), New Delhi for providing a fellowship under the BASE program (IUSSTF BASE Internships 2018/ 12/ Vignesh M, dt. 09/04/2018).

\section{References}

1. B. O'Regan and M. Gratzel, Nature, 1991, 353, 737-740.

2. M. Wang, N. Chamberland, L. Breau, J. E. Moser, R. HumphryBaker, B. t. Marsan, S. M. Zakeeruddin and M. Gratzel, Nat. Chem., 2010, 2, 385.

3. L. G. P. S., T. H. Nok, Y. Chenyi, Z. S. M., G. T. Michael and D. P. J., ChemSusChem, 2015, 8, 255-259.

4. R. Boonsin, J. Sudchanham, N. Panusophon, P. Sae-Heng, C. Sae-Kung and P. Pakawatpanurut, Mater. Chem. Phys., 2012, 132, 993-998.

5. K. C. Sun, I. A. Sahito, J. W. Noh, S. Y. Yeo, J. N. Im, S. C. Yi, Y. S. Kim and S. H. Jeong, J. Mater. Chem. A, 2016, 4, 458-465.

6. L. Tao, Z. Huo, S. Dai, J. Zhu, C. Zhang, X. Pan, Y. Huang, S. Yang, B. Zhang and J. Yao, Mater. Chem. Phys., 2015, 152, 6268.

7. F. Li, J. R. Jennings, X. Wang, L. Fan, Z. Y. Koh, H. Yu, L. Yan and Q. Wang, J. Phys. Chem. C, 2014, 118, 17153-17159.

8. A. S. Shaplov, R. Marcilla and D. Mecerreyes, Electrochim. Acta, 2015, 175, 18-34.

9. A. Ejigu, K. R. J. Lovelock, P. Licence and D. A. Walsh, Electrochim. Acta, 2011, 56, 10313-10320.

10. N. Papageorgiou, Y. Athanassov, M. Armand, P. Bonhote, H. Pettersson, A. Azam and M. Griatzel, J. Electrochem. Soc., 1996, 143, 3099-3108.

11. S. Malaisamy, P. Priyanka and M. Paramasivam, J. Appl. Polym. Sci., 2014, 131.

12. K. Prabakaran, S. Mohanty and S. K. Nayak, New J. Chem., 2015, 39, 8602-8613.

13. M. Fathy, A. B. Kashyout, J. El Nady, S. Ebrahim and M. B. Soliman, Alexandria Eng. J., 2016, 55, 1737-1743.

14. N. Pavithra, A. M. Asiri and S. Anandan, J. Power Sources, 2015, 286, 346-353.

15. M. A. K. L. Dissanayake, H. K. D. W. M. N. R. Divarathne, C. A. Thotawatthage, C. B. Dissanayake, G. K. R. Senadeera and B. M. R. Bandara, Electrochim. Acta, 2014, 130, 76-81.

16. R. A. Senthil, J. Theerthagiri, J. Madhavan and A. K. Arof, Opt. Mater, 2016, 58, 357-364.

17. S. K. Ahn, T. Ban, P. Sakthivel, J. W. Lee, Y. S. Gal, J. K. Lee, M.-R. Kim and S.-H. Jin, ACS Appl. Mater. Interfaces, 2012, 4, 2096-2100.

18. Y. Fang, W. Xiang, X. Zhou, Y. Lin and S. Fang, Electrochem. Commun., 2011, 13, 60-63.

19. S. H. Park, D. H. Won, H. J. Choi, W. P. Hwang, S. i. Jang, J. H. Kim, S. H. Jeong, J. U. Kim, J. K. Lee and M. R. Kim, Sol Energy Mater Sol Cells, 2011, 95, 296-300.

20. E. Vijayakumar, A. Subramania, Z. Fei and P. J. Dyson, J. Appl. Polym. Sci., 2015, 132, 42032.

21. S. Venkatesan, N. Hidayati, I. P. Liu and Y. L. Lee, J. Power Sources, 2016, 336, 385-390.

22. J. U. Kim, S. H. Park, H. J. Choi, W. K. Lee, J. K. Lee and M. R. Kim, Sol Energy Mater Sol Cells, 2009, 93, 803-807.

23. A. R. S. Priya, A. Subramania, Y. S. Jung and K. J. Kim, Langmuir, 2008, 24, 9816-9819.

24. S. Malaisamy, P. Priyanka and M. Paramasivam, J. Appl. Polym. Sci., 2014, 131.

25. W. W. Cui, D. Y. Tang, Z. 1. Gong and Y. D. Guo, J. Mater. Sci., 2012, 47, 6276-6285.

26. N. H. Ming, S. Ramesh and K. Ramesh, Sci. Rep., 2016, 6, 27630.

27. C. C. Yang, J. Y. Wey, T. H. Liou, Y. J. Li and J. Y. Shih, Mater. 
Chem. Phys., 2012, 132, 431-437.

28. B. Lin, T. Feng, F. Chu, S. Zhang, N. Yuan, G. Qiao and J. Ding, RSC Adv., 2015, 5, 57216-57222.

29. E. Vijayakumar, A. Subramania, Z. Fei and P. J. Dyson, RSC Adv., 2015, 5, 52026-52032.

30. Z. Huo, L. Tao, L. Wang, J. Zhu, S. Chen, C. Zhang, S. Dai and B. Zhang, Electrochim. Acta, 2015, 168, 313-319.

31. N. Zebardastan, M. H. Khanmirzaei, S. Ramesh and K. Ramesh, Electrochim. Acta, 2016, 220, 573-580.

32. V. Murugadoss, N. Wang, S. Tadakamalla, B. Wang, Z. Guo and S. Angaiah, J. Mater. Chem. A, 2017, 5, 14583-14594.

33. G. P. S. Lau, J. D. Decoppet, T. Moehl, S. M. Zakeeruddin, M. Gratzel and P. J. Dyson, Sci. Rep., 2015, 5, 18158.

34. H. Ozawa, YukiTawaraya and H. Arakawa, Electrochim. Acta, 2015, 151, 447-452.

35. T. Oncsik, A. Desert, G. Trefalt, M. Borkovec and I. Szilagyi, Phys. Chem. Chem. Phys., 2016, 18, 7511-7520.

36. Y. Bai, Y. Cao, J. Zhang, M. Wang, R. Li, P. Wang, S. M. Zakeeruddin and M. Griatzel, Nat. Mater., 2008, 7, 626.
37. C. T. Li, C. P. Lee, M. S. Fan, P. Y. Chen, R. Vittal and K. C. Ho, Nano Energy, 2014, 9, 1-14.

38. V. Elayappan, V. Murugadoss, S. Angaiah, Z. Fei and P. Dyson, J. Appl. Polym. Sci., 2015, 132, 42777.

39. S. Peng, L. Li, H. Tan, M. Srinivasan, S. G. Mhaisalkar, S. Ramakrishna and Q. Yan, Electrochim. Acta, 2013, 105, 447454.

40. X. Zong, K. Kim, D. Fang, S. Ran, B. S. Hsiao and B. Chu, Polym., 2002, 43, 4403-4412.

41. A. K. Solarajan, V. Murugadoss and S. Angaiah, Sci. Rep., 2017, 7, 45390 .

42. J. R. Macdonald, Annals of Biomedical Engineering, 1992, 20, 289-305.

43. A. M. Stephan, T. P. Kumar, M. A. Kulandainathan and N. A. Lakshmi, J. Phys. Chem. B, 2009, 113, 1963-1971.

44. A. K. Solarajan, V. Murugadoss and S. Angaiah, J. Appl. Polym. Sci., 2017, 134, 45177.

45. A. Solarajan, V. Murugadoss and S. Angaiah, Appl. Mater. Today, 2016, 5, 33-40. 